Komplementärmedizin

Li Tian, Anton Lachner

\section{Wortschatz Chinesische Medizin}

Die Sprache der TCM - verstehen, begreifen, korrekt aussprechen

München: Urban \& Fischer; 2005

478 Seiten, mit CD-ROM, kartoniert

Fr. 77.- / € 49.95

ISBN 978-3-437-55221-2

Wer beginnt, sich mit chinesischer Medizin zu beschäftigen oder Akupunktur zu erlernen, stösst schnell auf verschiedene chinesische Begriffe. Oft sind deutsche Übersetzungen oder entsprechende Bezeichnungen nicht bedeutungsgleich. Daher lohnt es sich auch für diejenigen, die nicht gleich die chinesische Sprache erlernen wollen, einen kleinen Teil des chinesischen Wortschatzes zu beherrschen. In verschiedenen Büchern werden die Begriffe leider nicht immer einheitlich und eindeutig verwendet. Oft werden sogar in ein und demselben Werk unterschiedliche Übersetzungen statt konsequent ein Begriff benutzt. Das kann schnell zur Verwirrung führen und zum Drang danach, die wahre Bedeutung zu verstehen, wobei dieses Buch sehr hilfreich ist.

Der Koautor Anton Lachner, der als Dozent im Lehr- und Forschungsbereich Chinesisch an der Universität Bern tätig ist, hat den gesamten ersten Teil, eine «Einführung in die chinesische Sprache», verfasst. Dort geht er ausführlich auf die Schriftzeichenetymologie, Wortbildung und Phonetik des Chinesischen ein. Diese erste Begegnung mit dem Chinesischen trägt zum besseren Verständnis bei, um tiefgründig die Chinesische Medizin und Heilmittelkunde studieren zu können.

Der zweite, der eigentliche Wortschatzteil wurde von Li Tian zusammengestellt. Sie hat nicht nur mit diesem Werk zur Verbreitung, Popularisierung und Verbesserung der Behandlung nach Traditioneller Chinesischer Medizin in Europa beigetragen. In ihrem Buch hat sie alle für die TCM relevanten Begriffe systematisch in 13 Kapitel geordnet. In chinesisch werden die Schriftzeichen und die Pinyinschreibung angegeben, und dazu wird

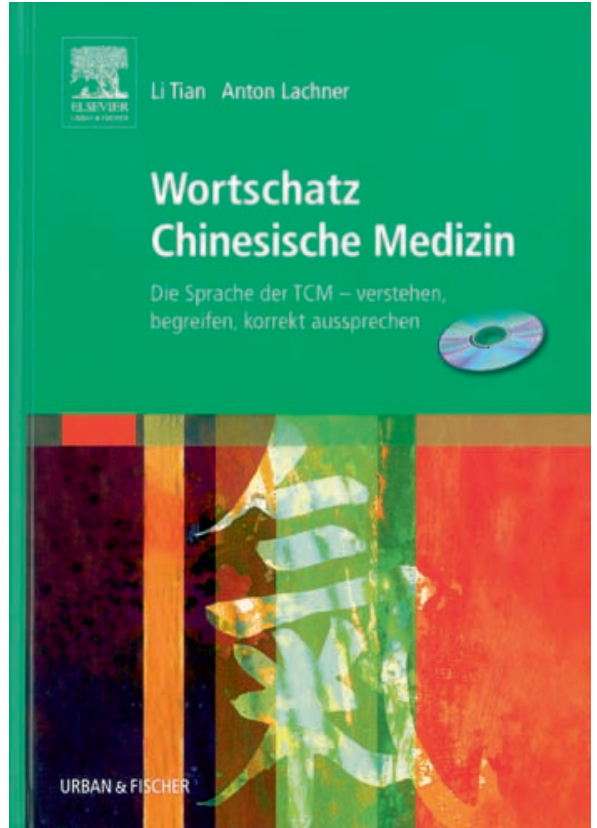

nicht nur die deutsche, sondern auch die englische Übersetzung aufgeführt, was sich bei der Verwendung englischer Literatur als nützlich erweist. Zum Erlernen der korrekten Aussprache kann man sich alle verwendeten Vokabeln auf der beiliegenden CD-ROM anhören. Im Kapitel «Akupunkturpunkte» werden zusätzlich die Zusammenhänge zwischen den Namen eines Punktes und der Lokalisation oder Funktion gezeigt. Das Buch eignet sich nicht sehr zum Nachschlagen, man sollte sich eher mit einem bestimmten Kapitel beschäftigen und kann die zum Thema gehörigen Vokabeln lernen. Jedoch sind die Akupunkturpunkte nicht nur nach ihrer Lokalisation auf den Meridianen geordnet, sondern zusätzlich in weiteren Tabellen nach der Bedeutung ihres chinesischen Namens, was auch hilft, ihre Funktion zu erahnen. Dort findet man dann auch die Bedeutung der in vielen Punkten immer wieder auftauchenden Teilnamen. In manchen Kapiteln gibt es Platz mit Kästchen und Hilfslinien für Schreibübungen der chinesischen Schriftzeichen.

Sandra Krüger, Neuenburg 